\title{
Smac/DIABLO can predict postoperative delirium in elderly patients after cardiac surgery : A prospective nested case-control study
}

\author{
Xin Ding ${ }^{1}$, Zeping $\mathrm{Li}^{1}$, Lei Lei ${ }^{1}$, Muhuo $\mathrm{Ji}^{2}$, and Jianjun Yang ${ }^{1}$ \\ ${ }^{1}$ The First Affiliated Hospital of Henan University of CM \\ ${ }^{2}$ Children's Hospital of Nanjing Medical University
}

December 11, 2021

\begin{abstract}
Background:Postoperative delirium (POD) is a common complication following cardiac surgery. Mitochondrial injury, demonstrated by excessively activated oxidative stress and regulating cell apoptosis, has been reported to contribute to POD. [Mitophagy](https://www.geenmedical.com/article?id=34752757\&type=true), apoptosis, pyroptosis, ferroptosis had been known to play a critical role in degenerative nervous system diseases. However, the serum change of Smac/DIABLO in POD induced by cardiac surgery/anesthesia is still undetermined. We designed the study to determine the expression level of SMAC/DIABLO in POD patients. Methods:A nested case-control study was performed, including 21 POD patients and a matched group of 63 non-POD controls.
\end{abstract}

\section{Introduction}

Postoperative delirium(POD) is an acute disorder of attention and cognition in postoperative patients, more often observed in those aged 65 or older[1]. Previously, studies had shown that delirium was strongly associated with cardiac postoperative prognoses, such as perioperative atrial fibrillation(POAF), death within 30 days, postoperative cognitive dysfunction(POCD), and dementia[2-4]. The pathomechanism of delirium is mainly related to the neuroinflammatory reaction, oxidative stress, ischemia-reperfusion injury, neuronal aging, and cholinergic dysfunction[5,6]. The elderly who lack physiologic reserves, with multi-system chronic disease are more likely to suffer from POD following cardiac operation[7]. The significant association between mortality after cardiac surgery and POD makes that becoming increasingly concerned[8].

Oxidative stress had been proved to contribute to the pathogenesis of postoperative delirium. Mitochondria as the most fragile injured organelles to hypoxia, primarily take place morphological and functional changes, such as mitochondrial fission-fusion disorder, membrane potential decrease, proteins of mitochondrial intercristae efflux into the cytoplasm. Mitochondrial dysfunction, viewed as hallmarks of aging and disturbed intracellular oxidative phosphorylation, has been reported as an early pathogenic event of Alzheimer's disease and involved in the pathogenesis of delirium-like behavior in aged mice[9,10]. Mitochondria, not only provide the site for intracellular oxidative phosphorylation, but also involve in cell information transmission, apoptosis, and regulating cell growth and cycle. Under apoptosis stimulation, cytochrome c was firstly released from the intermembrane of mitochondria, in company with the second mitochondria-derived activator of caspase (Smac/DIABLO) and other mitochondria proteins[11]. Cytochrome c directly activates downstream a series of caspases to promote apoptosis, while Smac/DIABLO was binding to inhibitor apoptosis proteins(IAPs) by weakening their inhibitory activity and resulting in nuclear damage[12-13]. Research had shown that overproduction of reactive oxygen species(ROS) and overexpression of cytochrome c, Smac/DIABLO, and caspases were observed in the ischemic injury rats model[14]. The mitochondrial apoptotic pathway leads to caspase 
activation playing an essential role in the machinery of neuronal cell death[15]. Therefore, we hypothesized that Surgery/Anesthesia might stimulate apoptosis-regulated proteins release and activate downstream signaling pathways, thus promoting the occurrence and development of POD. This study aimed to detect the plasma concentration of cytochrome c and Smac/DIABLO at 24 hours postoperative, and evaluate the diagnostic efficiency of cytochrome c and Smac/DIABLO, whether these could be as new markers of detecting POD. The findings of this investigation may help identify new underlying mechanisms of POD.

\section{Materials \& Methods}

This study was conducted at the Department of Anesthesiology in the first affiliated hospital of Zhengzhou University in June 2021 and July 2021. The Bioethics Committee of Zhengzhou University approved the study (permission no.2021-KY-0293), and written informed consent from all subjects. Basic patient information and surgical information are obtained from The first affiliated Hospital of Zhengzhou University electronic records. We included 108 cardiac patients aged 65years or older who underwent elective coronary artery bypass graft or valve surgery or both and were admitted postoperative directly to the surgical intensive care unit. Exclusion criteria were: emergency procedure, preoperative combined with disturbance of consciousness, MMSE score below the lower limit of corresponding education background, suffering from malignant tumor, Long-term use of immunosuppressive agents or hormones, or refusal to provide written informed consent. The individual was excluded for MMSE score in the lower limit of corresponding education background, to eliminate the combined impairment of cognitive function before surgery. We excluded patients who suffered from a malignant tumor and Long-term use of immunosuppressive agents or hormones because the tumor will affect apoptosis proteins expression level to a great extent. Delirium was assessed with the Confusion Assessment Method for Intensive Care Unit patients at 12-hour intervals for postoperative three consecutive days. Each patient was examined for delirium before surgery (baseline) and then every 12 hours for three consecutive days, from the morning on the next day postoperative. The Confusion Assessment Method for the Intensive Care Unit (CAM ICU) was used to identify the presence or absence of delirium. CAM ICU is a validated and commonly used score to assess delirium in ICU patients. With CAM ICU, four features of delirium were assessed: 1) acute onset and a fluctuating course, 2) inattention, 3) disorganized thinking, and 4) an altered level of consciousness. To diagnose delirium, features 1, 2, and either 3 or 4 should be recognized as positive. As a part of CAM ICU evaluation, the Richmond AgitationSedation Scale(RASS) is used to assess altered levels of consciousness and fluctuations of mental status. All physicians involved in the study were trained on how to perform the CAM ICU delirium screening. Patients were divided into delirium group or non-delirium group after the three days follow-up record. We collected arterial blood samples in $24 \mathrm{~h}$ postoperative and quantitative analysis of plasma concentration of Cytochrome C, Smac/Diablo, AIF, and caspase 3(boster,Zhengzhou,china) by ELISA assay kit. Thereafter, we analyzed the differents of expression levels in two groups and calculated areas under the ROC curve for predicting postoperative delirium.

Statistical analysis was performed using SPSS 21.0 software. Data statistics were expressed as the mean \pm standard deviation median and interquartile range(IQR) for continuous variables, and frequencies and percentages for discrete variables. Mann-Whitney U test was used to evaluate continuous data that does not fit a normal distribution. $\mathrm{P}$ values $<0.05$ were considered statistically significant. Odds ratios (ORs) $95 \%$ confidence intervals (CIs) were calculated depending on the plasma concentration compared to $43.2 \mathrm{pg} / \mathrm{ml}$, the cut-off was determined by ROC curve analysis and YouDen index. The sample size was calculated using PASS 15.0 software. According to pre-experiment results the Smac/Diablo predictive value for 48 patients is 0.75 , we set power 0.9 , alpha 0.05 . We verified the sample size by finally statistical results, there are enough power to demonstrate our results. The significance criterion of 0.05 and all tests were 2 -sided. Group allocation was set 3 for the ratio of the non-delirium group to delirium group, based on the incidence of delirium during our follow-up. We picked and quantitatively analyzed the blood sample of patients who presented with typical clinical manifestation, such as hyperactive delirium with manic status requiring the use of physical restraints, hypoactive delirium with lethargy/inattentiveness/apathy, excluded patients who developed suspicious 
symptoms or might be in incubation period.

\section{Results}

\section{Clinical characteristics}

Out of 108 patients included in the study, three patients were excluded because of death, one patient was excluded because of renal failure needs dialysis. Among the 104 patients, 21 (20.2\%) developed postoperative delirium (group POD $(+)$ ) and 83 did not (group POD (-)). Moreover, nine patients were excluded because of failing to collect blood samples in postoperative 24h. Finally, 21 patients were in group POD $(+)$; meanwhile, 63 matched group POD (-) patients were selected in a ratio of 1:3 based on propensity scores using nearestneighbor matching. The propensity score was calculated based on age, sex, and operation time. Baseline demographic and perioperative characteristics of the PSM cohort were well balanced. The final analytic sample of 84 participants was obtained (as shown in figure 1 ). The characteristics of the study population are shown in Table $\mathbf{1}$. There were no significant differences between groups in factors related to the patient's clinical condition assessed with the NYHA classification, EuroSCORE II, and Charlson Comorbidity Index(CCI). There was no statistical difference between the study groups in the incidence of comorbidities such as acute coronary syndromes(ACS), hypertension, lung disease, history of atrial fibrillation, diabetes mellitus, cerebral vascular disease, or chronic kidney disease(CKD). There was no statistical difference between the study groups in the incidence of history of percutaneous coronary intervention(PCI). There were no significant differences in preoperative serum index, such as Hb, cTn, BNP, Scr(Table 1). There were no significant differences between groups in factors related to the operation: the ratio of cardiopulmonary bypass in both groups, mean aortic cross-clamping time in CBP patients, mean hemoglobin level, mean lactic acid level. Mechanical ventilation time in the POD $(+)$ group was longer than patients in the POD (-) group $(\mathrm{p}=0.047)$. The ICU length of stay in the POD $(+)$ group was longer than patients in the POD (-) group $(\mathrm{p}=0.015)$.

\section{Figure1.Demographic characteristics of the study population.}

\section{Table 1. Comparison of clinical characteristic parameters.}

Continuous variables data are presented as mean $\pm \mathrm{SD}$, data that does not satisfy a normal distribution are presented as Median (interquartile range); Count data are presented as number and percentage [N, (\%)].

\section{Comparison of delirium incidences and serological indicators of patients in POD $(+)$ and POD (-) groups}

The Confusion Assessment Method for the Intensive Care Unit (CAM ICU) was conducted after surgery. Each patient was examined for delirium before surgery (baseline) and then every 12 hours for three consecutive days, starting in the morning on the first day after surgery. There were 10 cases $(47.6 \%)$ of all delirium episodes that occurred on the first postoperative day, 5 cases $(23.8 \%)$ on the second, and five cases $(23.8 \%)$ on the third day. Except, one patient was conscious and cooperative for three days postoperative, and sudden onset of disturbance of consciousness in the sixth postoperative day and transferred into ICU. We recorded and included this case in the $\mathrm{POD}(+)$ group.

Arterial blood samples were collected in $24 \mathrm{~h}$ postoperative, and a total of 84 samples were analyzed. The plasma concentration of Smac/Diablo in the POD $(+)$ group was 39.7 (interquartile range [IQR],36.3-45.2), lower than that in the POD (-) group was 48.9(interquartile range[IQR],44.3-60.1). The plasma concentration of Cyto-c in the POD (+) group was 17.9 (interquartile range [IQR],14.6-21.1) and had no significant differences with the POD (-) group (18.8, interquartile range[IQR],16.3-20.5). The mean plasma concentration of AIF and caspase 3 in $\operatorname{POD}(+)$ was lower than $\operatorname{POD}(-)$, but was not statistically significant. To determine the sensitivity and specificity of Smac/DIABLO predicting the occurrence of POD, we performed ROC analysis (Figure 2). The area under the ROC curve (AUC) of Smac/DIABLO used to predict POD was 0.81(95\% CI: $0.71-0.89, \mathrm{p}=0.0001)$. Based on the ROC curve analysis and Youden Index, the optimal cut-off value for Smac/DIABLO was $43.2 \mathrm{pg} / \mathrm{ml}$, and the sensitivity and specificity were $81.0 \%$ and $71.4 \%$, respectively (Table 3). We divided the study population into two groups according to the cut-off value of Smac/DIABLO. 
Individuals plasma concentration of Smac/DIABLO lower than $43.2 \mathrm{pg} / \mathrm{ml}$ in $24 \mathrm{~h}$ postoperative were more likely to have signs of delirium[odds ratio $(\mathrm{OR})=10.6,95 \%$ confidence interval (CI): 3.4-33.1, $\mathrm{p}=0.000$ ].

\section{Figure 2.ROC curve of Smac/DIABLO for prodicting POD DISCUSSION}

Delirium is an acute neurological complication that frequently occurred in elderly people after orthopedic or cardiac surgery as the reserve becomes inadequate to cover the metabolic requirements and organs more fragile to severe oxidative stress[16]. Previously, a study shows that $21.4 \%(384 / 1,797)$ of patients above 65 years developed POD following cardiac surgery, and 33.5\% (77/230) in octogenarians[17], higher than that in patients aged $<65$ years that range from $2.1 \%$ to $9.4 \%[18]$. Some identifiable risk factors had been approved associated with POD such as old age, comorbid illness, major trauma, and baseline dementia. It is of great significance for preoperative identity and postoperative treatment of delirium and improving the prognosis of patients.

Our study showed that $20.2 \%$ of patients aged [?]65 years experienced delirium after cardiac surgeries. We used the Confusion Assessment Method for the Intensive Care Unit (CAM ICU) for the identification of delirium. Theoretically, The CAM ICU scale be conducted for serial delirium assessments at least 3-day by a trained researcher or clinicians[1]. We examined for delirium in each patient before surgery (baseline) and then every 12 hours for three consecutive days. However, one patient suddenly onset of disturbance of consciousness and soon afterward in a coma on the sixth postoperative day during our follow-up. The patient was transferred into ICU immediately, consciousness recovered and discharged after symptomatic treatment. Recently, Hamadnalla $\mathrm{H}$ et al. [19] study suggested that compared to delirium assessments twice daily for five days, twice daily assessments for 4 days detected an estimated 97\% (95\% CI: 91\%-99\%) of delirium and twice daily for three days detected $90 \%$ (95\% CI:82\%-95\%) of delirium.

Mitochondria, as the major organelles that produce energy in the cell, was named "powerhouse". In general, mitochondrial function is impaired and release toxic proteins when apoptotic stimuli act on cells, manifests as decreased ATP supply, overproduced reactive oxygen species (ROS), and a series of cysteine-aspartate proteases (caspases) activation[9,20]. Recently, mitochondrial fusion/fission dynamics imbalance contributes to delirium-like behavior in aged mice has been reported[21]. Mitochondrial DNA haplogroup has been verified that is associate with the development of and pathogenesis of delirium during sepsis[22]. Thus, we conducted this study to explore whether intrinsic (mitochondrial) apoptosis has an association with postoperative delirium. That pathway has been widely reported in the tumor, represents potential targets for inhibitors in cancerous cells [23,24]. In proteins that fall off from mitochondrial intermembrane into the cytosol, cytochrome c was the first protein molecule to be transferred into the cytosol and the second mitochondria-derived activator of caspase/direct IAP-binding protein with low PI(Smac/DIABLO) were transferred soon afterward. Both of these proteins have been shown to contribute to caspase-independent cell death processes[25]. Accompanied with other mitochondrial proteins, such as the serine protease AIF, HtrA2/OMI (high-temperature requirement protein A2), endonuclease G efflux from mitochondria. The other proteins were released into the cytosol and launched nuclear DNA damage or instigate cytotoxicity (Figure 3) [26]. Mizutani Y et al. [27] firstly analyze circulating levels of Smac/DIABLO in the serum and suggest that the levels of Smac/DIABLO in the serum may be considered a valid biomarker in patients with different stages of bladder cancer. Our study shows that the median plasma concentration of Smac/DIABLO in the group of $\operatorname{POD}(+)$ patients was significantly lower than that in $\operatorname{POD}(-)$ patients in $24 \mathrm{~h}$ postoperative. ROC curve analysis to evaluate the predictive value of Smac/DIABLO for POD in elderly patients and found that the AUC is 0.81 and the optimal cut-off value for Smac/DIABLO was $43.2 \mathrm{pg} / \mathrm{ml}$.

The concentration of Cytochrome c, AIF, and caspase 3 has no significant difference in the two groups. This is not what we expected Springs S et al. [28] observed that the velocity for Smac efflux from the mitochondria of cells subjected to staurosporine-induced apoptosis is approximately four times longer than the time for cytochrome c efflux, that may suggest Smac and cytochrome c may efflux the mitochondria by different pathways. 
Smac/DIABLO is widely expressed in most tissues and organs, synthesized in the nucleus, and does not act in normal tissue[29]. The elevated levels of Smac/DIABLO in the circulation might have resulted from its release following apoptosis of cells. In contrast, the low serum levels of Smac/DIABLO in the serum may reflect dysfunctional cell escape from apoptosis process without cleaning damaged organelles and proteins timely and replaced by normal cells[26]. Autophagy and apoptosis are sequential progress within cells that control the turnover of organelles and proteins. According to the degree of stress, protein pathways elicit various degrees of autophagy and apoptosis within the same cell. Generally, autophagy and apoptosis are mutually antagonistic systems,apoptosis-associated pathway activation shuts off the autophagic process and vice versa. However, autophagy in some cases may also participate in cell death, and has been viewed as a separate modality of programmed cell death (PCD)[30,31]. When ischemia and hypoxia dwindle nutrient supply, the autophagy system will be activated and generally considered a protective response. From this perspective, we suppose that a lower level of Smac/DIABLO in POD patients signifies a greater degree of autophagy system activation. The precise underlying mechanism remains unclear.

The heart is the directly target organ during cardiac surgery and mostly suffers from ischemia, while the brain is the most fragile organ to ischemia and hypoxia. Ji X et al. build an ischemia-reperfusion injury model by inserting a guidewire into the ventricle to induce ventricular fibrillation. By isolating brain mitochondria and cardiac mitochondrial sub-populations and comparing calcium retention capacity, ADP stimulated oxygen consumption, ETC activity to evaluate mitochondrial function after injured. Their results showed that brain mitochondria are far more sensitive to ischemia injury during cardiac arrest compared to cardiac mitochondria[32]. So we suppose that Smac/DIABLO levels in circulation and the association with delirium is a not chance finding.

There are limitations to our study. We only detect of concentration of these proteins at one-time point, and our target proteins act at different stages in the pathological process of POD[13,33]. Further systematic studies are needed to explore the variation trend of apoptosis-related proteins. The study was deliberately limited to elderly patients, a population at a higher risk of suffering from postoperative delirium, and did not analyze the association of Smac/DIABLO and cytochrome c with POCD, dementia and Long term mortality. Additional research conducted on a larger population, and an examination of long-term complications will shed light on the question of the predictive value of Smac/DIABLO for long-term consequences after cardiac surgery. This study is designed as a pilot study, and further study requires a separate second dataset to identify if the results remain valid.

In recent years, studies have shown that mitochondrial dysfunction was one of the factors involved in cerebral ischemia-reperfusion injuries, so mitochondria are viewed as an attractive target in neuroprotection. Mitochondrial-targeted therapy of the nervous system gradually started from fundamental study to clinical application. Several novel therapeutic agents, such as cyclosporine A, Nicorandilan, and Mitochondrial calcium uniporter (MUC) by adjust the mitochondrion channel, reducing oxidative stress and Ca2+ overload, inhibiting ROS burst, protect nerve cells[34,35]. Further researches on these mitochondrial-targeted drugs might provide an available strategy for future POD treatment.

In summary, Smac/DIABLO in elderly patients, which are significantly related to the incidence of acute delirium following cardiac surgery, is a predictable biomarker for POD. Postoperative serum levels evaluation can help identify POD patients and provide a clinical basis for the primary prevention of POD in elderly patients.

The figure 3 was refer to the website of Cell Signaling Technology (CST) and Chan P, et al. research [36].

\section{CONCLUSIONS:}

These results demonstrate that plasma concentration of Smac/Diablo in the next day postoperative might be a sensitive index for predicting postoperative delirium in older cardiac surgical patients. Further studies should be conducted to verify the role of Smac/DIABLO in the development of POD.

\section{Acknowledgements}




\section{Author Contribution}

Xin Ding, MD:

This author helped conceive the manuscript, perform the literature review, write the full text, edit subsequent drafts, and designed all tables and figures.

\section{Zeping Li, MD:}

This author helped review the manuscript, edit and modify drafts.

\section{Lei Lei, MD:}

This author helped review the manuscript, edit and modify drafts.

Muhuo Ji, MD, PhD:

This author helped review the manuscript, edit and modify drafts.

Jianjun Yang, MD, PhD:

This author helped design the study, review the article, and review and approve the final manuscript.

This manuscript was handled by: Jianjun Yang.

The authors acknowledge funding support from the National Natural Science Foundation of China(81971020).

The study were approved by the Bioethics Committee of Zhengzhou University (permission no.2021-KY0293).

The authors declare no conflicts of interest.

All authors read and approved the final manuscript.

Written informed consent has been obtained from the patients to publish this paper.

REFERENCES

1. Naeije G, Pepersack T. Delirium in elderly people. Lancet (London, England). 2014; 383: 2044-5.

2. Witlox J, Eurelings L, et al. Delirium in elderly patients and the risk of postdischarge mortality, institutionalization, and dementia: a meta-analysis. JAMA. 2010; 304:443-51.

3. MacLullich A, Shenkin S, et al. The 4 'A's test for detecting delirium in acute medical patients: a diagnostic accuracy study. Health technology assessment (Winchester, England). 2019; 23: 1-194.

4. Davis D, Muniz Terrera G, et al. Delirium is a strong risk factor for dementia in the oldestold: a population-based cohort study. Brain: a journal of neurology. 2012; 135: 2809-16.

5. Lopez M, Pandharipande $P$, et al. Intraoperative cerebral oxygenation, oxidative injury, and delirium following cardiac surgery. Free radical biology \& medicine. 2017; 103: 192-8.

6. Maldonado J. Neuropathogenesis of delirium: review of current etiologic theories and common pathways. The American journal of geriatric psychiatry : official journal of the American Association for Geriatric Psychiatry. 2013; 21: 1190-222.

7. Ansaloni L, Catena F, et al. Risk factors and incidence of postoperative delirium in elderly patients after elective and emergency surgery. The British journal of surgery. 2010; 97: 273-80.

8. Gottesman R, Grega $M$, et al.Delirium after coronary artery bypass graft surgery and late mortality. Annals of neurology. 2010; 67: 338-44. 
9. Van der Rijt S, Molenaars M, et al. Integrating the Hallmarks of Aging Throughout the Tree of Life: A Focus on Mitochondrial Dysfunction. Frontiers in cell and developmental biology. 2020; 8: 594416.

10. Palikaras K, Achanta K, et al. elegansAlteration of mitochondrial homeostasis is an early event in a model of human tauopathy. Aging. 2021; 13.http://10.18632/aging.203683.PMID:34751671.

11. Mootha V, Wei M, et al. A reversible component of mitochondrial respiratory dysfunction in apoptosis can be rescued by exogenous cytochrome c. The EMBO journal. 2001; 20: 661-71.

12. Du C, Fang M, et al. Smac, a mitochondrial protein that promotes cytochrome c-dependent caspase activation by eliminating IAP inhibition. Cell. 2000; 102: 33-42.

13. Vega-Naredo I, Oliveira G, et al. Analysis of Proapoptotic Protein Trafficking to and from Mitochondria. Methods in molecular biology (Clifton, NJ). 2021; 2310: 161-78.

14. Cheng Z, Mi X, et al. [IMMP2L gene mutation activates mitochondrial apoptotic pathway to aggravate cerebral ischemic injury in mice]. Xi bao yu fen zi mian yi xue za zhi $=$ Chinese journal of cellular and molecular immunology. 2021; 37: 616-22.PMID:34140073.

15. Sugawara $T$, Noshita $N$, et al. Overexpression of copper/zinc superoxide dismutase in transgenic rats protects vulnerable neurons against ischemic damage by blocking the mitochondrial pathway of caspase activation. The Journal of neuroscience : the official journal of the Society for Neuroscience. 2002; 22: 209-17.

16. Bugiani $O$. Why is delirium more frequent in the elderly? Neurological sciences : official journal of the Italian Neurological Society and of the Italian Society of Clinical Neurophysiology. 2021.

17. Kotfis K, Szylińska A, et al. Early delirium after cardiac surgery: an analysis of incidence and risk factors in elderly ([?]65 years) and very elderly ([?]80 years) patients. Clinical interventions in aging. 2018; 13: 1061-70.

18. Duan X, Coburn M, et al. Efficacy of perioperative dexmedetomidine on postoperative delirium: systematic review and meta-analysis with trial sequential analysis of randomised controlled trials. British journal of anaesthesia. 2018; 121: 384-97.

19. Hamadnalla $H$, Sessler D, et al. Optimal interval and duration of CAM-ICU assessments for delirium detection after cardiac surgery. Journal of clinical anesthesia. 2021; 71: 110233.

20. Robertson J, Orrenius S. Role of mitochondria in toxic cell death. Toxicology. 2002: 491-6.

21. Lu Y, Chen L, et al.Surgery/Anesthesia disturbs mitochondrial fission/fusion dynamics in the brain of aged mice with postoperative delirium. Aging. 2020; 12: 844-65.

22. Samuels D, Hulgan T, et al.Mitochondrial DNA Haplogroups and Delirium During Sepsis. Critical care medicine. 2019; 47: 1065-71.

23. Li K, van Delft M, et al. Too much death can kill you: inhibiting intrinsic apoptosis to treat disease. The EMBO journal. 2021:e107341.

24. Mao L, Liu H, et al. PINK1/Parkin-mediated mitophagy inhibits warangalone-induced mitochondrial apoptosis in breast cancer cells. Aging. 2021; 13: 12955-72.

25. Cregan S, Dawson V, et al. Role of AIF in caspase-dependent and caspase-independent cell death. Oncogene. 2004; 23: 2785-96. 
26. Saelens X, Festjens N, et al. Toxic proteins released from mitochondria in cell death. Oncogene. 2004; 23: 2861-74.

27. Mizutani Y, Katsuoka Y, et al. Low circulating serum levels of second

mitochondria-derived activator of caspase (Smac/DIABLO) in patients with bladder cancer. International journal of oncology. 2012; 40:1246-50

28. Springs S, Diavolitsis V, et al. The kinetics of translocation of Smac/DIABLO from the mitochondria to the cytosol in HeLa cells. The Journal of biological chemistry. 2002; 277: 45715-8.

29. Adrain C, Creagh E, et al. Apoptosis-associated release of Smac/DIABLO from mitochondria requires active caspases and is blocked by Bcl-2. The EMBO journal. 2001; 20: 6627-36.

30. Denton D, Nicolson S, et al. Cell death by autophagy: facts and apparent artefacts. Cell death and differentiation. 2012; 19: 87-95.http://10.1038/cdd.2011.146.PMID:22052193.

31. Mariño G, Niso-Santano M. Self-consumption: the interplay of autophagy and apoptosis. Nature reviews Molecular cell biology. 2014; 15: 8194.http://10.1038/nrm3735.PMID:24401948.

32. Ji X, Bradley J, et al, Shabnam R, Peberdy M, Ornato J, Chen Q, Lesnefsky E, Tang W. Cerebral and myocardial mitochondrial injury differ in a rat model of cardiac arrest and cardiopulmonary resuscitation. Biomedicine \& pharmacotherapy = Biomedecine \& pharmacotherapie. 2021; 140: 111743.http://10.1016/j.biopha.2021.111743.PMID:34020243

33. Shen L, Gan Q, et al. Mitophagy in Cerebral Ischemia and Ischemia/Reperfusion Injury. Frontiers in aging neuroscience. 2021; 13: 687246.

34. Okahara A, Koga J, et al. Simultaneous targeting of mitochondria and monocytes enhances neuroprotection against ischemia-reperfusion injury. Scientific reports. 2020; 10: 14435.

35. Chen Y, Guo S, et al. Mitochondrial Fusion and Fission in Neuronal Death Induced by Cerebral Ischemia-Reperfusion and Its Clinical Application: A Mini-Review. Medical science monitor : international medical journal of experimental and clinical research. 2020; 26: e928651.

36. Chan P. Mitochondria and neuronal death/survival signaling pathways in cerebral ischemia. Neurochemical research. 2004; 29: 1943-9. 


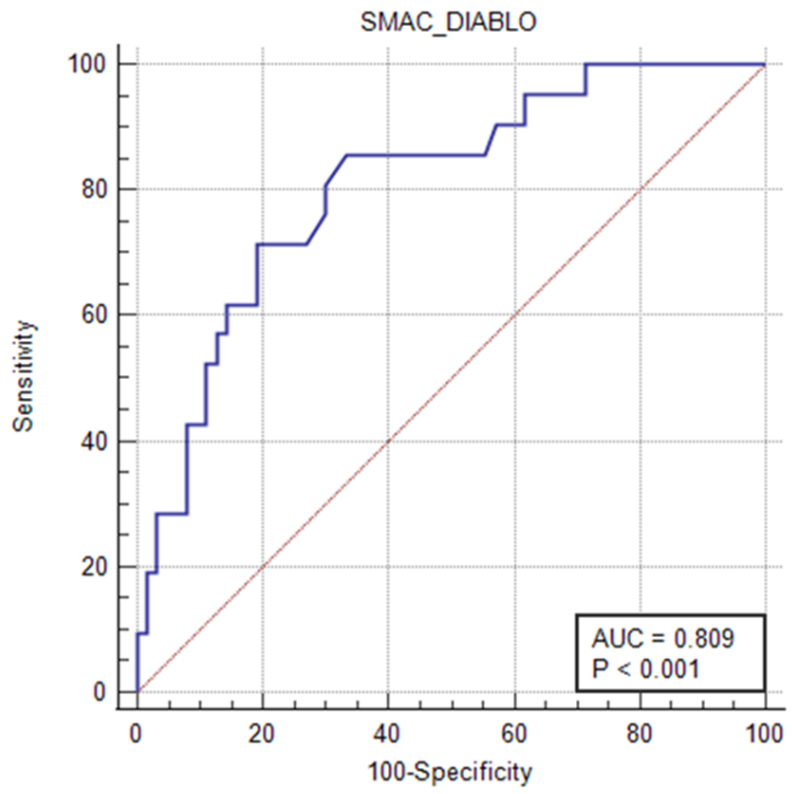




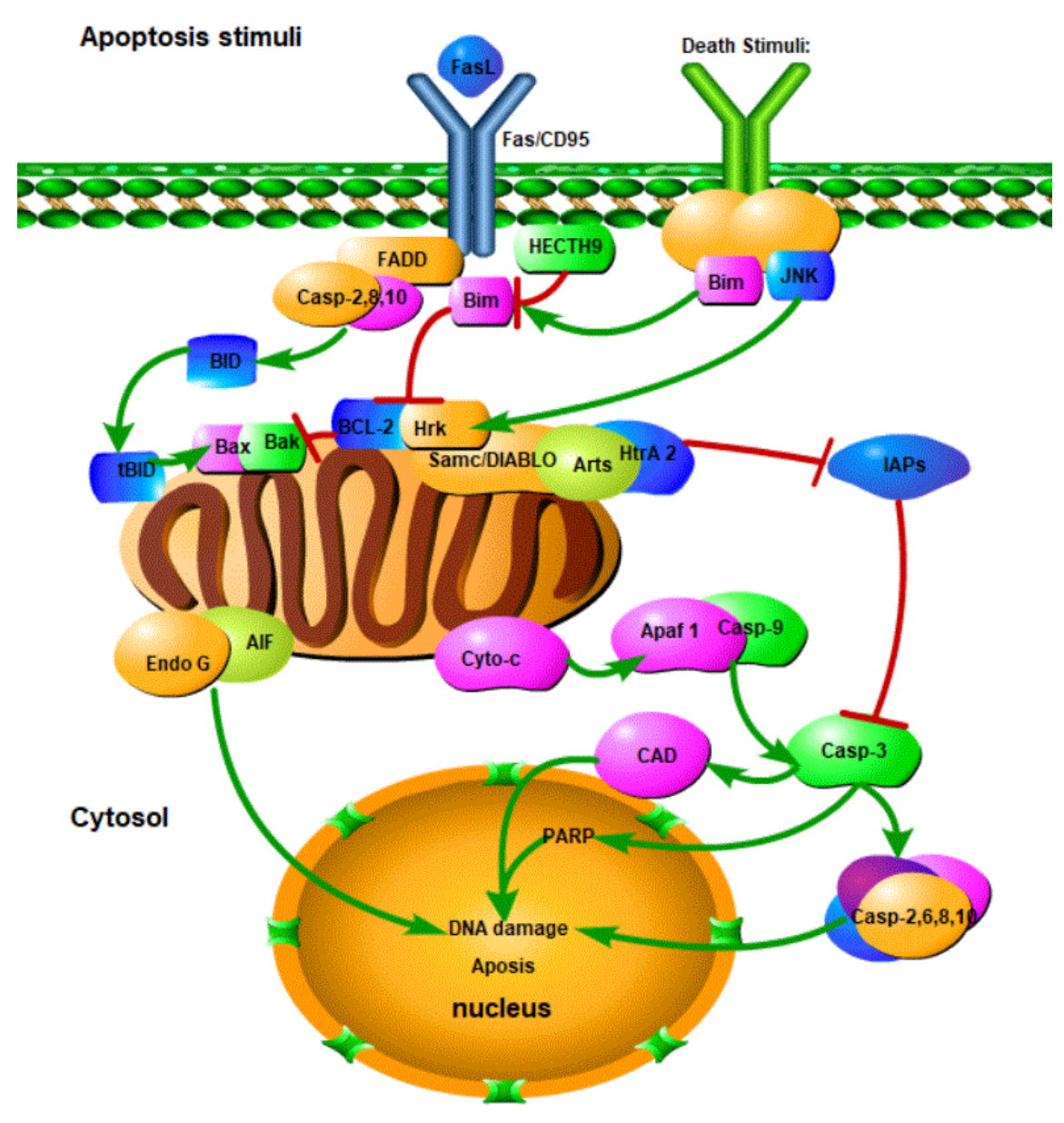




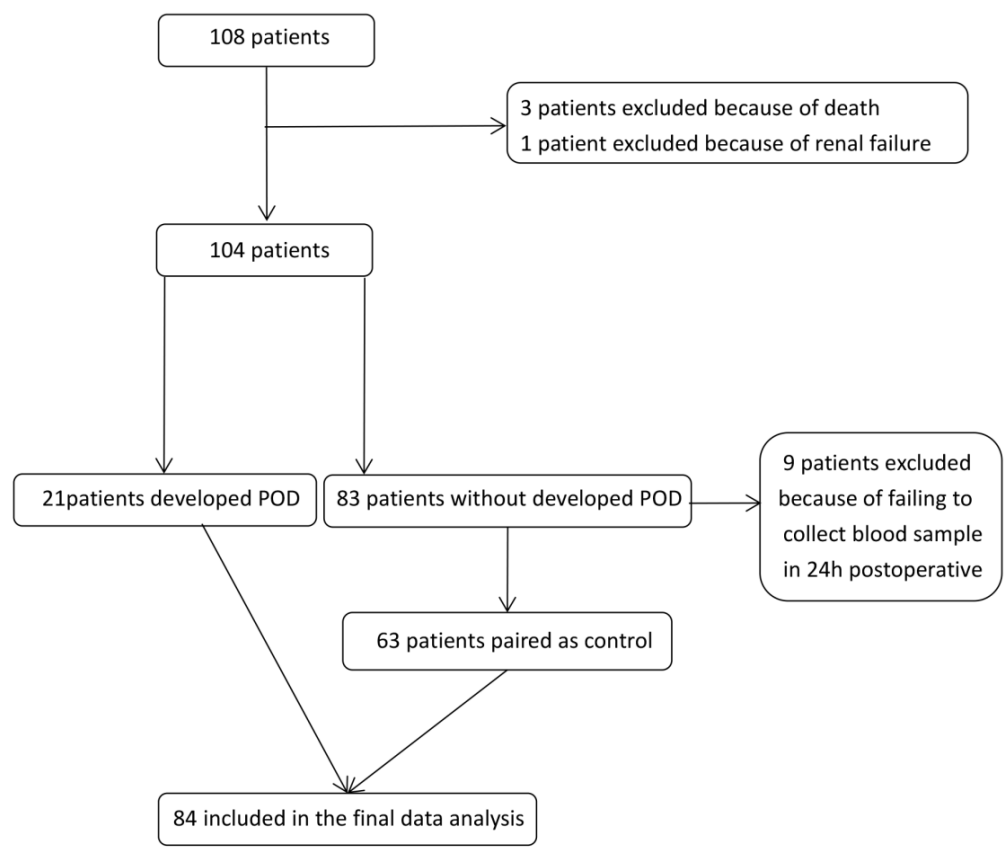

\section{Hosted file}

table1peerj.docx available at https://authorea.com/users/450701/articles/549001-smacdiablo-can-predict-postoperative-delirium-in-elderly-patients-after-cardiac-surgerya-prospective-nested-case-control-study

\section{Hosted file}

table2peerj.docx available at https://authorea.com/users/450701/articles/549001-smacdiablo-can-predict-postoperative-delirium-in-elderly-patients-after-cardiac-surgerya-prospective-nested-case-control-study

\section{Hosted file}

table3peerj.docx available at https://authorea.com/users/450701/articles/549001-smacdiablo-can-predict-postoperative-delirium-in-elderly-patients-after-cardiac-surgerya-prospective-nested-case-control-study 